\title{
The Potential of a Stroke Registry Using Diagnosis Procedure Combination Data from All Hospitals in a Japanese Prefecture
}

\author{
Shinichi Omama ${ }^{a}$ Kozo Tanno ${ }^{b}$ Yoshihiro Inoue $^{a}$ Kuniaki Ogasawara ${ }^{c}$

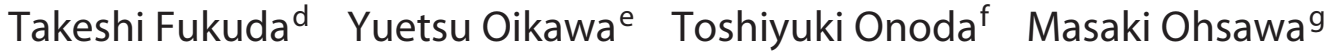 \\ Kiyomi Sakatab \\ a Iwate Prefectural Advanced Critical Care and Emergency Center, Iwate Medical University, Yahaba, Japan; \\ ${ }^{b}$ Department of Hygiene and Preventive Medicine, Iwate Medical University, Yahaba, Japan; 'Department of \\ Neurosurgery, Iwate Medical University, Yahaba, Japan; dDepartment of Neurosurgery, Iwate Prefectural Ninohe \\ Hospital, Ninohe, Japan; 'Department of Internal Medicine, Magokoro Hospital, Oshu, Japan; ${ }^{f}$ Health Service Center, \\ Iwate University, Morioka, Japan; ${ }^{9}$ Department of Internal Medicine, Morioka Tsunagi Onsen Hospital, Morioka, Japan
}

\section{Keywords}

Cerebrovascular disease $\cdot$ Stroke registry $\cdot$ Diagnosis

procedure combination $\cdot$ Incidence rate

\begin{abstract}
Objectives: In Japan, many hospitals have joined the diagnosis procedure combination/per-diem payment system (DPC/PDPS), which provides unified information about inpatients. DPC data are digitized, and the number of participating hospitals has increased recently. Herein, we evaluated the potential of a stroke registry constructed using these unified DPC data from all hospitals in the Iwate Prefecture, Japan. Methods: The proportion of cerebrovascular disease (CVD) cases registered by DPC-participating hospitals was calculated and compared with all registered cases in the Iwate Stroke Registry in 2008-2017. The cases were categorized based on sex, age-groups, stroke subtypes, and firstever onset or recurrence onset. Based on the registered cases in the stroke registry, the accuracy of the CVD cases extracted by the disease name from DPC data of a typical core hospital and a typical noncore hospital was evaluated. $\boldsymbol{R e}$ -
\end{abstract}

sults: Of the 71 hospitals with 9,992 beds in the Iwate Prefecture in 2018, 50 hospitals with 8,316 beds participated in the DPC system. The proportion of registered cases from participating hospitals was $95.2 \%(44,779 / 47,018)$ for all stroke types (95.6\% men and $94.9 \%$ women), $94.3 \%$ for cerebral infarction, $97.0 \%$ for intracerebral hemorrhage, and $98.7 \%$ for subarachnoid hemorrhage, whereas it was $95.7 \%$ for firstever onset and $94.1 \%$ for recurrent onset. The proportion of registered cases decreased with increasing patient age. Attending doctors and researchers registered 486 and 41 CVD cases from the core and noncore hospitals, respectively, whereas 455 and 46 CVD cases were extracted from the DPC data of these hospitals, respectively. This yielded $86.6 \%$ sensitivity, $99.3 \%$ specificity, $92.5 \%$ positive predictive value, and $98.7 \%$ negative predictive value for the core hospital; these values were $92.7 \%, 98.6 \%, 82.6 \%$, and $99.5 \%$, respectively, for the noncore hospital. Discussion/Conclusions: The stroke registry constructed using DPC data from all hospitals of Iwate Prefecture appears to be adequately complete and accurate.

(C) 2022 The Author(s)

Published by S. Karger AG, Basel karger@karger.com www.karger.com/ced

Karger ${ }^{\prime \prime} \div$

BOPEN ACCESS
(C) 2022 The Author(s)

Published by S. Karger AG, Basel

This is an Open Access article licensed under the Creative Common Attribution-NonCommercial-4.0 International License (CC BY-NC) (http://www.karger.com/Services/OpenAccessLicense), applicable to the online version of the article only. Usage and distribution for commercial purposes requires written permission.
Correspondence to:

Shinichi Omama, somama@iwate-med.ac.jp 


\section{Introduction}

In Japan, population-based national mortality rates show that cerebrovascular disease (CVD) has descended to the fourth place among causes of death, but it remains the most common disease in the elderly who need longterm care $[1,2]$. Previously, population-based national incidence rates of CVD in the Japanese were not unknown because relevant registries or prevalence statistics were not available. A health insurance system for all citizens, which is paid in proportion to the total medical treatment fee, is in place since 1961 in Japan. In complement, the diagnosis procedure combination/per-diem payment system (DPC/PDPS) of Japan for acute injuries or illness is a prospective payment system that uses a classification code based on disease diagnosis and aims to standardize medical care, similar to the diagnosis-related groups/prospective payment system. It was launched as part of the health insurance system in 2003 [3-5]. Under DPC, data on personal identification, name of injury and/ or illness, and medical treatment of the patient are submitted to the Health, Labor, and Welfare Ministry of Japan for reimbursement of medical and/or technical service rendered. Recently, the number of hospitals that have either joined or are submitting data to the DPC/PDPS has increased rapidly because participating hospitals receive additional medical service fees from the health insurance system [3]. Furthermore, treatment cost for almost all CVD patients is reimbursed by the health insurance system; thus, a CVD registry using DPC data from all hospitals represents a potentially usable substitute to a population-based CVD registry. To verify the usefulness of DPC data, this study evaluated the completeness and accuracy of a CVD registry built using available DPC data from all hospitals.

\section{Materials and Methods}

\section{Completeness of DPC Data}

The Iwate Stroke Registry, with all hospitals and other medical facilities, clinics, and healthcare facilities, has been jointly operated by the government of the Iwate Prefecture and the Iwate Medical Association since 1991. The stroke diagnostic criteria of cerebral infarction (CEI), intracerebral hemorrhage (ICH), and subarachnoid hemorrhage (SAH) in this registry are based principally on the criteria established for the Monitoring System for Cardiovascular Disease commissioned by the Ministry of Health and Welfare [6]. These criteria correspond to the International Classification of Disease version 10 (ICD-10) code published by the World Health Organization, which defines stroke as a sudden onset of neurological symptoms [7]. All data in the stroke registry were verified by well-trained staff for inac- curacy. Retrospective registration from death certificates was not performed as per the regulations of the Iwate Stroke Registry. An inventory survey of the registry was conducted from January 1,2008 , in most hospitals with beds for general patients in the Iwate Prefecture and in core hospitals in the neighboring areas that treat acute phase CVD patients. This survey assigned trained researchers, including specialty physicians, nurses, or health information managers, to those hospitals. Details of the Iwate Stroke Registry and its inventory survey have been reported previously $[8,9]$. In this study, "DPC facilities" were defined as those that participated in DPC/PDPS and non-DPC/PDPS facilities that submitted data to the DPC till April 2018; other hospitals or medical facilities were termed "non-DPC facilities." Information on CVD cases between January 1, 2008, and December 31, 2017, was acquired from the Iwate Stroke Registry, and was based on the day of occurrence. The proportion of CVD cases registered at the Iwate Stroke Registry from DPC facilities was determined based on all registered cases and was categorized by sex, age-group (under 35, 35-44, 45-54, 55-64, 65-74, $75-84,85-94$, and $\geq 95$ years), stroke subtypes (CEI, ICH, or $\mathrm{SAH})$, and as first-ever onset or recurrent onset.

\section{Accuracy of Disease Name in DPC Data}

The DPC database provides clinical information on the inpatient, including unique identification data, date of birth, sex, residential postal code, date of admission and discharge, outcome at discharge, and disease or injury names with their ICD-10 codes $[3,7]$. Disease names and their ICD-10 codes are listed for 6 items, namely, "main illness," "illness leading to hospitalization," "illness with highest medical cost," "illness with second-highest medical cost," "comorbid diseases at admission," and "diseases occurring after admission." CVD names and their ICD-10 codes in this study were defined as follows: SAH, I60; ICH, I61; and CEI, I63 [7], and suspected CVD names were not included. For the analysis of CVD disease name accuracy in DPC data from each facility, DPC data were used from both DPC/PDPS and non-DPC/PDPS facilities.

The Iwate Prefectural Ninohe Hospital is a core hospital that has joined the DPC/PCPS and has 253 beds for general patients with regular attending neurologists and neurosurgeons. Cases registered with the disease name "CVD" on any of the 6 items described above or their combinations were extracted for the period between April 1, 2016, and March 31, 2018. Sensitivity, specificity, positive predictive value (PPV), and negative predictive value (NPV) of the extracted cases from DPC data were evaluated based on cases registered in the Iwate Stroke Registry that were diagnosed by the attending physicians and research nurses during the study period.

Magokoro Hospital is a noncore hospital that has not joined the DPC/PDPS but submitted data to DPC during sampling. It has 48 beds for general patients without regular CVD specialty. Using the best combination of disease names listed in the DPC data of the Ninohe Hospital, the sensitivity, specificity, PPV, and NPV of the CVD cases extracted from DPC data of Magokoro Hospital between April 1, 2020, and March 31, 2021, were evaluated based on the cases registered in the Iwate Stroke Registry by a research doctor during the inventory survey of the registry during the same period. The IBM SPSS Statistics 20.0 (IBM Corp., Armonk, NY, USA) software was used for accuracy analyses. 
Table 1. Number of the registered cases and age at onset of cerebrovascular diseases in DPC facilities and non-DPC facilities: data from the Iwate Stroke Registry

\begin{tabular}{|c|c|c|c|c|c|c|}
\hline \multirow[t]{2}{*}{ Sex } & \multicolumn{2}{|l|}{ DPC facilities } & \multicolumn{2}{|c|}{ Non-DPC facilities } & \multicolumn{2}{|l|}{ All facilities } \\
\hline & cases, $n(\%)$ & age (SD) & cases, $n(\%)$ & age (SD) & cases, $n(\%)$ & age (SD) \\
\hline Men & 23,549 (95.6) & $71.3(12.4)$ & $1,087(4.4)$ & $74.7(11.3)$ & $24,636(100)$ & $71.4(12.4)$ \\
\hline Women & 21,230 (94.9) & $77.6(12.4)$ & $1,152(5.1)$ & $82.0(9.3)$ & $22,382(100)$ & $77.8(12.3)$ \\
\hline Total & $44,779(95.2)$ & $74.3(12.8)$ & $2,239(4.8)$ & $78.4(11.0)$ & $47,018(100)$ & 74.5 (12.8) \\
\hline
\end{tabular}

DPC, diagnosis procedure combination/per-diem payment system in Japan; DPC facilities, the facilities which participated in DPC; non-DPC facilities, the facilities which do not participate in DPC; cases, the number of the registered cases; age, the mean age at onset of cerebrovascular diseases; SD, standard deviation.

\section{Results}

\section{Completeness of DPC Data}

From the first year of DPC/PDPS (2003), the number of DPC facilities in the Iwate Prefecture has increased (Fig. 1), and in 2018, 70.4\% (50/71) of all hospitals with beds for general patients and $83.4 \%(8,316 / 9,992)$ of all beds for general patients were DPC facilities. The number of registered cases at Iwate Stroke Registry between April 1, 2008, and December 31, 2017, along with age at onset of CVD from DPC facilities and non-DPC facilities, is listed in Table 1. Cases from DPC facilities accounted for 95.2\% of all registered cases, and patients from DPC facilities were younger than those from non-DPC facilities. Data on the number of registered cases and the proportion of the cases from DPC facilities and non-DPC facilities by age-groups are depicted in Figure 2. The proportion of cases registered at DPC facilities decreased as patient age increased, among both men and women (Fig. 2c, d). The proportion of cases registered at DPC facilities was $94.3 \%$ for CEI, $97.0 \%$ for $\mathrm{ICH}$, and $98.7 \%$ for $\mathrm{SAH}$, while it was $95.7 \%$ for first-ever onset and $94.1 \%$ for recurrent onset.

\section{Accuracy of Disease Name in DPC Data}

The number of cases registered in the DPC from the Iwate Prefectural Ninohe Hospital for the study period was 5,433 , while 486 cases were registered at the Iwate Stroke Registry by an attending physician or research nurse during the same period. Table 2 provides data on the number of cases extracted based on disease name lists in DPC data, and the sensitivity, specificity, PPV, and NPV of these extracted cases based on the registered cases by an attending physician and research nurse. Cases extracted for the items "main illness," "illness leading to hospitalization," and "illness with highest medical cost" showed high sensitivity, PPV, and NPV, respectively. Cas-

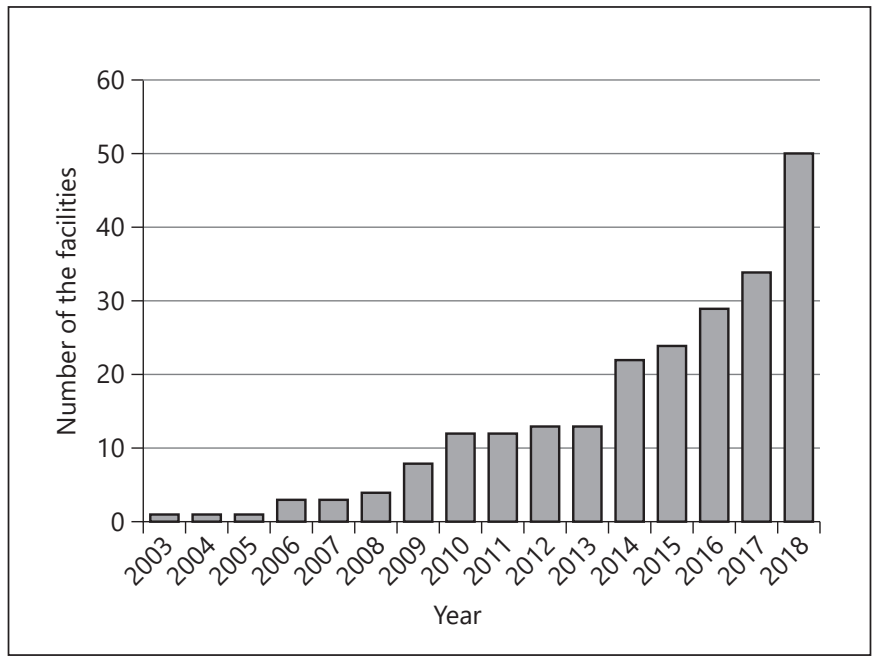

Fig. 1. Number of hospitals participating in the DPC/PDPS in the Iwate Prefecture. DPC/PDPS, diagnosis procedure combination/ per-diem payment system.

es extracted using the combination of the above 3 items and "illness with second-highest medical cost" had higher sensitivity than the cases extracted using the 3 disease name lists, but showed comparable specificity and PPV.

For the Magokoro Hospital, DPC data were available for 614 cases, and 41 cases were registered by a research doctor during inventory survey in the same period. The number of the CVD cases extracted from DPC data using the combination "main illness," "illness leading to hospitalization," "illness with highest medical cost," and "illness with second-highest medical cost" was 46 cases, and sensitivity, specificity, PPV, and NPV for these cases, calculated based on the cases registered at the Iwate Stroke Registry by research doctors, were $92.7 \%, 98.6 \%, 82.6 \%$, and $99.5 \%$, respectively. 

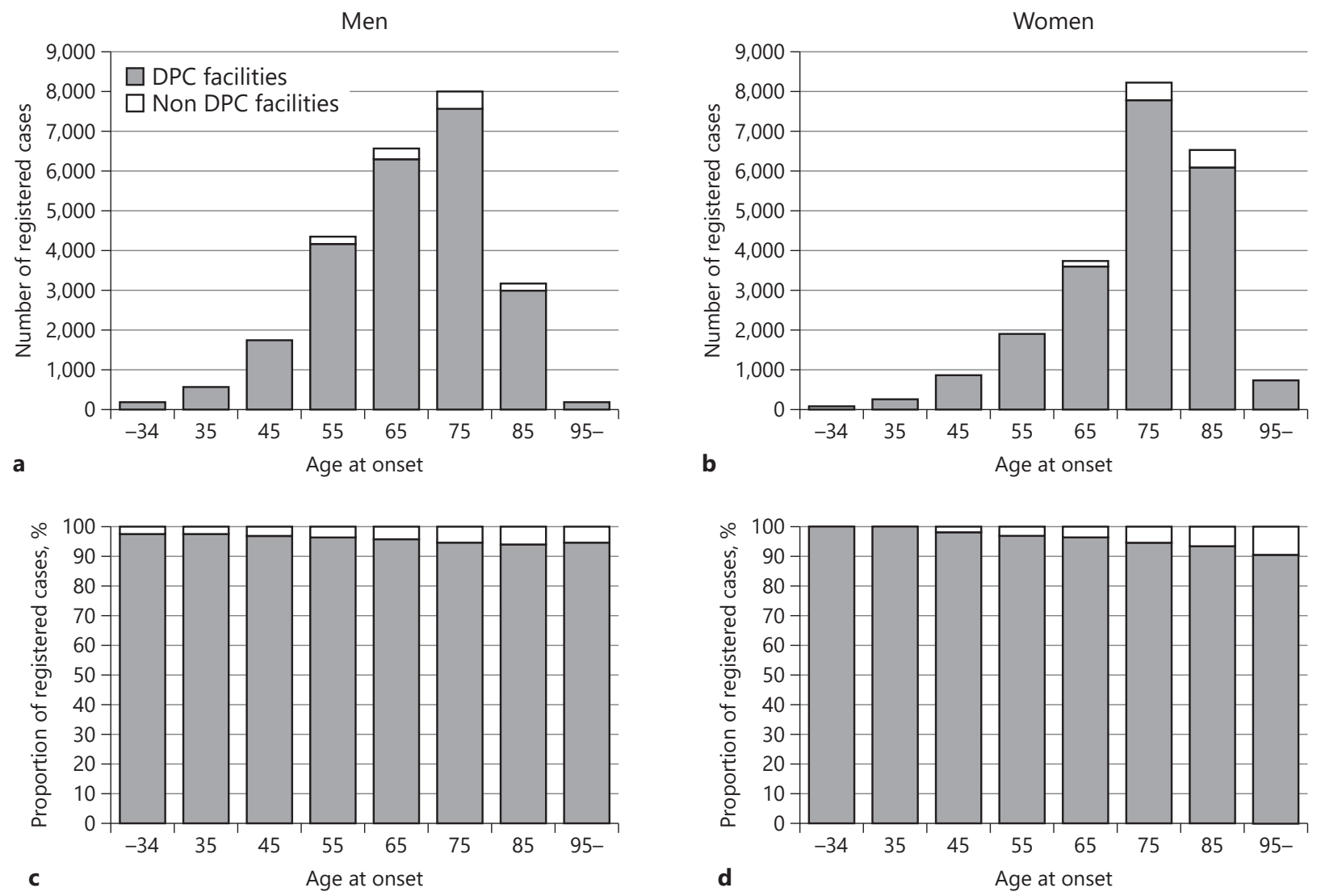

Fig. 2. Age-group-based categorization of the number and proportion of cases from the hospital registered at DPC hospitals and the facilities not participating in DPC. Filled bar shows DPC facilities, and empty bar shows non-DPC facilities. Panel a shows the number of registered cases of men. Panel $\mathbf{b}$ shows the number of the registered cases of women. Panel c shows the proportion of registered cases of men. Panel $\mathbf{d}$ shows the proportion of registered cases of women. DPC, diagnosis procedure combination.

Table 2. Accuracy of the disease name in DPC data

\begin{tabular}{|c|c|c|c|c|c|c|}
\hline A. The main disease & 441 & 417 & 85.8 & 99.5 & 94.6 & 98.6 \\
\hline B. The disease leading to hospitalization & 427 & 406 & 83.5 & 99.6 & 95.1 & 98.4 \\
\hline C. The disease of highest medical cost & 444 & 419 & 86.2 & 99.5 & 94.4 & 98.7 \\
\hline D. The disease of second highest medical cost & 27 & 18 & 3.7 & 99.8 & 66.7 & 91.3 \\
\hline E. The comorbid diseases at admission & 119 & 48 & 9.9 & 98.6 & 40.3 & 91.8 \\
\hline$A$ and $B$ and $C$ & 448 & 419 & 86.2 & 99.4 & 93.5 & 98.7 \\
\hline$A$ and $B$ and $C$ and $D$ & 455 & 421 & 86.6 & 99.3 & 92.5 & 98.7 \\
\hline$A$ and $B$ and $C$ and $E$ & 518 & 422 & 86.8 & 98.1 & 81.5 & 98.7 \\
\hline$A$ and $B$ and $C$ and $F$ & 595 & 419 & 86.2 & 96.4 & 70.4 & 98.6 \\
\hline
\end{tabular}

DPC, diagnosis procedure combination; PPV, positive predictive value; NPV, negative predictive value. 


\section{Discussion/Conclusion}

We evaluated the usefulness and completeness of data available in a CVD registry that used DPC data from all facilities that participated in the DPC/PDPS program and from facilities that submitted relevant data to the DPC. Our results show that the available data are adequately complete.

\section{Completeness of DPC Data}

Japan has a rapidly growing proportion of the elderly, and as the number and proportion of the elderly who suffer from CVD are predicted to increase, the social and familial burden of long-term care is also expected to increase $[2,8]$. The rate of CVD and its trend, especially among the elderly, are important indexes of the medical care system and cost of nursing care of the Japanese healthcare policy, at both the national and local levels. In Japan, patients who suffer from CVD receive treatment at not only core hospitals but also noncore hospitals, and noncore hospitals often treat elderly CVD patients [9]. Thus, as hospital-based CVD registries are often constructed with core hospitals alone, they would have fewer elderly patients than expected due to this selection bias $[10,11]$. Completeness of a disease registry is also influenced by the level of cooperation from participating facilities. Voluntary cooperation for a disease registry can lead to fewer registrations due to inadvertent mistakes, noncooperation owing to disinterest, or the labor-intensive process of registration. An inventory survey for a disease registry can effectively achieve and maintain a high degree of completeness by preventing nonregistration, but it is labor-intensive and requires cooperation from each participating facility $[8,12,13]$. Thus, a simplified registration process would be desirable when constructing a disease registry that relies on inputs from many hospitals, including noncore hospitals. Hospitals participating in the DPC/PDPS program or those submitting DPC data must provide relevant data regularly, for example, monthly, to request reimbursement of treatment fee. If the cases for a disease registry are extracted from DPC data and their registry forms are made based on DPC data, the burden of registration on the staff would be greatly reduced, which enable the registry to achieve and maintain sufficient completeness.

\section{Accuracy of the Disease Name of DPC Data}

Previous studies have evaluated the accuracy of the term "CVD" in DPC data and have reported 50.0\% sensitivity, $98.9 \%$ specificity, $86.4 \% \mathrm{PPV}$, and $93.5 \% \mathrm{NPV}$
[14]; however, we report higher sensitivity and PPV. A potential reason for such higher sensitivity may be the fact that DPC data were checked by both attending physicians and medical coding staff in both core and noncore hospitals of this study. Nevertheless, about $10 \%$ of the CVD cases were missed in 1 core hospital, probably due to omission of the input term "CVD" in DPC and/or no reimbursement requested from the DPC/PDPS in certain cases, for example, death in the emergency room or out of hospital or hospital stay of $<24 \mathrm{~h}$. The high PPV of cases extracted from DPC data at the core hospital is attributed to the fact that it is the only core hospital in that medical district that predominantly treats acute illness or trauma with few chronic patients. PPV of cases at the noncore facility is slightly lower and is ascribed to multiple chronic illness or disease names provided in the DPC data.

\section{Limitations}

This study has a few limitations. First, the accuracy of disease names in DPC data from other unsurveyed hospitals remains unknown, and constructing a CVD registry using DPC data from all hospitals requires validation of the accuracy of DPC data from each hospital. Next, detailed information on history of illness and medication, onset time, admission time, start time of treatment or surgery, and imaging findings is not included in DPC data, and a CVD registry extracted from DPC data by disease name is suitable only for simple analyses, such as incidence of CVD. Comprehensive analyses of CVD will need hospital-based registration with detailed information, for which core hospitals with CVD specialists are more suitable. Nonetheless, a combination of population- or DPC-based simple CVD registry and a core hospital-based detailed CVD registry is desirable for estimating the true prevalence of CVD among the Japanese.

We did not evaluate the completeness and accuracy of other disease names in DPC data. If the accuracy of identifying other disease names in DPC data was as high as that in CVD, the DPC data may serve as a general disease registry.

\section{Acknowledgments}

Special thanks to Dr. Naohiko Kubo, who is the chief of the committee of the Iwate Stroke Registry, and Ms. Maki Kawakami, Ms. Kyoko Kanno, and Ms. Eiko Ikeda who are the staff of the Iwate Stroke Registry. We dedicate this work to late Dr. Kazuyoshi Itai, who was the staff of the study group of this work. 


\section{Statement of Ethics}

Informed consent for the registered patients of the Iwate Stroke Registry is not required because the registry is conducted by the government of Iwate Prefecture and the information of the registry is publicly available on the website of the Iwate Medical Association. The information and the point of contact for patients opt out of the inventory survey of the Iwate Stroke Registry and this study were announced on the message board for the public in the hospitals which joined the survey and this study. Usage of the Iwate Stroke Registry data for this study was approved by the Ethics Committees of the Iwate Stroke Registry (approval no. R2-1). This study was approved by the Ethics Committees of the Iwate Prefectural Ninohe Hospital, Magokoro Hospital, and Iwate Medical University, School of Medicine (approval No. MH2019-074).

\section{Conflict of Interest Statement}

The authors have no conflicts of interest to declare.

\section{Funding Sources}

This study was supported by JSPS KAKENHI, Grant No. JP19K10627, and the commissioned research of Iwate Prefecture.

\section{Author Contributions}

S.O. involved in concept, design, acquisition, analysis, interpretation, and drafting. T.K. was responsible for concept, design, analysis, interpretation, and supervision. T.F., Y.O., and M.O. performed acquisition and interpretation. T.O. involved in data management and supervision. Y.I., K.O., and K.S. were responsible for supervision and final approval of the manuscript.

\section{Data Availability Statement}

The data supporting the findings of this study are not publicly available due to the regulation of the Iwate Stroke Registry, which prohibits release of the registry data.

\section{References}

1 Health, Labour and Welfare Ministry of Japan. Population survey report. 2020. Available from: https://www.mhlw.go.jp/toukei/ saikin/hw/jinkou/geppo/nengai20/index. html Accessed on 2021 Oct 2.

2 Cabinet Officer. White paper on aging society. 2020. Available from: https://www8.cao. go.jp/kourei/whitepaper/w-2020/html/zenbun/index.html Accessed on 2021 Oct 2.

3 Hayashida K, Murakami G, Matsuda S, Fushi$\mathrm{mi}$ K. History and profile of diagnosis procedure combination (DPC): development of a real data collection system for acute inpatient care in Japan. J Epidemiol. 2021;31(1):1-11.

4 Ikegami N, Yoo BK, Hashimoto H, Matsumoto $\mathrm{M}$, Ogata $\mathrm{H}$, Babazono $\mathrm{A}$, et al. Japanese universal health coverage: evolution, achievements, and challenges. Lancet. 2011;378: 1106-15.

5 Ishii M. DRG/PPS and DPC/PDPS as prospective payment systems. Japan Med Assoc J. 2012;55:279-91.
6 Ministry of Health and Welfare. Study project of monitoring system for cardiovascular disease commissioned by the Ministry of Health and Welfare: manual for the registry and follow-up of stroke. Osaka, Japan: National Cardiovascular Center; 1988.

7 World Health Organization. International statistical classification of diseases and related health problems. 2nd ed. Geneva: World Health Organization; 2003.

8 Omama S, Ogasawara K, Inoue $\mathrm{Y}$, Ishibashi $\mathrm{Y}$, Ohsawa M, Onoda T, et al. Ten-year cerebrovascular disease trend occurrence by population-based stroke registry in an aging Japan local prefecture. J Stroke Cerebrovasc Dis. 2020;29:104580.

9 Omama S, Ogasawara K, Ishibashi Y, Nakamura M, Tanno K, Sakata K, et al. The impact and effectivity of an inventory survey for a stroke registry in Iwate prefecture. J Stroke Cerebrovasc Dis. 2017;26:2160-6.

10 Cui R, Iso H, Yamagishi K, Saito I, Kokubo Y, Inoue $\mathrm{M}$, et al. Trends in the proportions of stroke subtypes and coronary heart disease in the Japanese men and women from 1995 to 2009. Atherosclerosis. 2016;248:219-23.
11 Kimura K, Kazui S, Minematsu K, Yamaguchi T; Japan Multicenter Stroke Investigator's Collaboration. Anaysis of 16,922 patients with acute ischemic stroke and transient ischemic attack in Japan. A hospital-based prospective registration study. Cerebrovasc Dis. 2004; 18:47-56.

12 Takashima N, Arima H, Kita Y, Fujii T, Miyamatsu N, Komori M, et al. Incidence, management and short-term outcome of stroke in a general population of 1.4 million Japanese Shiga Stroke Registry. Circ J. 2017;81:163646.

13 Kubo M, Kiyohara Y, Kato I, Tanizaki Y, Arima H, Tanaka K, et al. Trends in the incidence, mortality, and survival rate of cardiovascular disease in a Japanese community. The Hisayama study. Stroke. 2003;34:234954.

14 Yamana H, Moriwaki M, Horiguchi H, Kodan M, Fushimi K, Yasunaga H. Validity of diagnoses, procedures, and laboratory data in Japanese administrative data. J Epidemiol. 2017;27:476-82. 